\title{
The Kovacs effect in model glasses
}

\author{
E.M. Bertin ${ }^{1}$, J.-P. Bouchaud ${ }^{1}$, J.-M. Drouffe ${ }^{2}$, C. Godrèche ${ }^{1,3}$ \\ ${ }^{1}$ Service de Physique de l'État Condensé, CEA Saclay, F-91191 Gif-sur-Yvette Cedex, France \\ 2 Service de Physique Théorique, CEA Saclay, F-91191 Gif-sur-Yvette Cedex, France \\ 3 Dipartimento di Fisica, Università di Roma "La Sapienza" and Center for Statistical Mechanics and Complexity, \\ INFM Roma 1, Piazzale AldoMoro 2, I-00185 Roma, Italy
}

\begin{abstract}
We discuss the 'memory effect' discovered in the 60's by Kovacs in temperature shift experiments on glassy polymers, where the volume (or energy) displays a non monotonous time behaviour. This effect is generic and is observed on a variety of different glassy systems (including granular materials). The aim of this paper is to discuss whether some microscopic information can be extracted from a quantitative analysis of the 'Kovacs hump'. We study analytically two families of theoretical models: domain growth and traps, for which detailed predictions of the shape of the hump can be obtained. Qualitatively, the Kovacs effect reflects the heterogeneity of the system: its description requires to deal not only with averages but with a full probability distribution (of domain sizes or of relaxation times). We end by some suggestions for a quantitative analysis of experimental results.
\end{abstract}

\section{INTRODUCTION. THE KOVACS EFFECT}

Systems with slow or glassy dynamics often exhibit non trivial behaviour when temperature changes are applied within the glassy phase. Since the system is out of equilibrium, one expects that its properties generically depend on the history of the system, an effect that is often called 'memory'. However, this general term embraces rather different effects. In the recent spin-glass literature, memory is associated to a two time observable, such as the a.c. susceptibility (that depends both on the frequency and on the age of the system) or any other response function. It has been shown that after a negative temperature cycle, the a.c. susceptibility recovers the exact value it had before the negative temperature jump, hence the name memory. This effect would be trivial if the dynamics was totally frozen at low temperature, whereas experiments show very clearly that some noticeable evolution in fact takes place [1-3]. The same qualitative effect, although not as clear-cut as in spin-glasses, has been observed in many other glassy materials (polymers, colloids, ferro-electrics, etc.) [4-8].

There is however another well known 'memory effect' that was discovered by Kovacs fourty years ago. This effect concerns one time observables, such as the specific volume, or the energy density, etc. and clearly shows that the non equilibrium state of the system cannot be fully characterized by the (time dependent) value of thermodynamical variables. The procedure followed by Kovacs was the following [9]: first, a reference curve is obtained by quenching the sample from a high temperature $T_{0}$ to a low temperature $T_{2}$, and measuring the time dependent volume $V(t)$ until a time $t_{e q}$ where the system can be considered to be in equilibrium. This defines a volume $V_{e q}\left(T_{2}\right)=V\left(t_{e q}\right)$. In a second step, the sample is quenched again from $T_{0}$ to a temperature $T_{1}<T_{2}$, until a certain time $t_{1}$. The temperature is then quickly raised from $T_{1}$ to $T_{2}$. The time $t_{1}$ is chosen such that the volume just after the jump reaches the value $V\left(t_{1}^{+}\right)=V_{e q}\left(T_{2}\right)$ - whereas in equilibrium $\left(t_{1} \rightarrow \infty\right)$ one would have $V_{e q}\left(T_{1}\right)<V_{e q}\left(T_{2}\right)$. Naively, one expects that nothing should happen, since the volume is already at its 'correct' equilibrium value $V_{e q}\left(T_{2}\right)$ at the new temperature. The volume $V(t)$ in fact shows a non monotonic behaviour for $t>t_{1}$, first increasing and then relaxing back to the equilibrium value $V_{e q}\left(T_{2}\right)$ :

$$
V(t)=V_{e q}\left(T_{2}\right)+\Delta V(t)
$$

where $\Delta V \geq 0$ is the 'Kovacs hump', such that $\Delta V\left(t=t_{1}^{+}\right)=0$ and $\Delta V(t \rightarrow \infty)=0$. Note that the condition $V\left(t_{1}^{+}\right)=V_{e q}\left(T_{2}\right)$ (and not $V\left(t_{1}^{-}\right)=V_{e q}\left(T_{2}\right)$ ) is chosen as to remove the trivial part of the effect, due to the thermal expansion of the fast (local) degrees of freedom. This subtlety in the Kovacs protocol is in fact quite important, as will be clear below.

Fig. 1 reproduces the original results published by Kovacs in 1963 [9], obtained on polyvinyl acetate. The Kovacs effect shows unambiguously that other 'internal' variables, beside the volume, are needed to characterize the out of equilibrium state of the system, and that these variables did not reach their $T_{2}$ equilibrium value at the end of the first stage. The memory in this case refers to the fact that these internal variables keep track, to some extent, of the system history. To avoid confusion between the different types of memory effects, we will follow [10] and call the above phenomenon the Kovacs effect. The Kovacs hump is characterized by its height $\Delta V_{K}$, and by the time $\tau_{K}$ for which the maximum is reached: $\Delta V\left(t=t_{1}+\tau_{K}\right)=\Delta V_{K}$. Qualitatively, the height $\Delta V_{K}$ grows with the temperature difference $T_{2}-T_{1}$ (it should obviously be zero for $T_{1}=T_{2}$ ), whereas the time $\tau_{K}$ decreases when $T_{2}-T_{1}$ increases. 


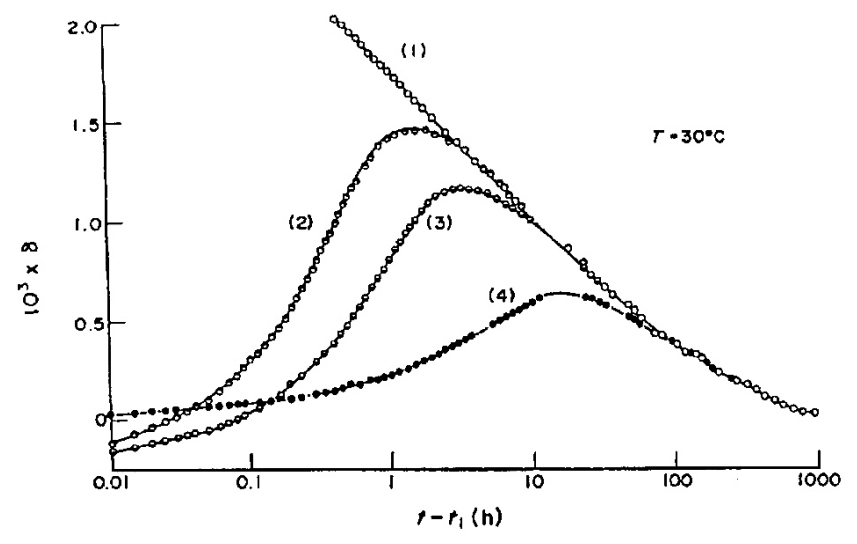

FIG. 1. Isothermal evolution at $T_{2}=30^{\circ} \mathrm{C}$ of the relative variation of the volume $\left(\times 10^{3}\right)$ in polyvinyl acetate: after a direct quench from $T_{0}=40^{\circ} \mathrm{C}$ to $T_{2}=30^{\circ} \mathrm{C}$ (1); after quenches from $T_{0}=40^{\circ} \mathrm{C}$ to $T_{1}=10^{\circ} \mathrm{C}$ (2), $15^{\circ} \mathrm{C}$ (3), or $25^{\circ} \mathrm{C}$ (4) followed by rapid re-heating at $T_{2}=30^{\circ} \mathrm{C}$. Data taken from A. J. Kovacs, Adv. Polym. Sci. 3, 394 (1963).

A similar effect was recently reported in the context of granular materials [11]. In the first stage of another type of experiment one 'taps' the system with three different amplitudes - say weak, moderate and strong- during a time chosen such as to reach a certain density, identical in the three cases. In the second stage of the experiment, the tapping amplitude is chosen to be moderate. The density just after the amplitude 'jump' is recorded. If the state of the system was only described by its density, the evolution of the density after the jump should be identical for all three situations, and follow the 'moderate' reference curve. This is not the case: as for the polymer glass, the weakly tapped system first has to dilate before it is able to resume its compaction, whereas the strongly tapped system compacts faster than the reference system just after the jump [11].

Finally, the same effect was recently observed in a numerical simulation of three dimensional spin-glasses [12] and in a realistic model of molecular liquid [13]. In spin-glasses, the energy density reveals the characteristic Kovacs hump when the temperature is raised ; the height of the hump and the time of the maximum behave qualitatively as in polymer glasses. Features similar to the Kovacs effect have also been identified experimentally in dipolar glasses [14] and spin glasses [15]. Since the Kovacs effect seems to be rather ubiquitous, a natural question is whether the underlying physics is the same in all these systems. Stated differently, can the effect select between different microscopic models of glassy dynamics?

The aim of this somewhat didactic paper is to discuss some simple models that allow to shed light on the above questions. In these models, the 'internal' variables referred to above appear as a whole distribution function (of domain sizes, or of relaxation times) of which only the mean is fixed by the experimental protocol, whereas the shape of the distribution keeps track of the system history. We show that the Kovacs effect is indeed rather generic, but that the detailed shape of the 'Kovacs hump' could reveal some useful microscopic information on the underlying glassy dynamics (see also the discussion in [16]). We first discuss models where slow dynamics is due to a coarsening mechanism, and recall and generalize the main results of [10]. We then turn to the Kovacs effect in the trap model, where detailed calculations can be performed. We end the paper with some suggestions for further analyzing experimental results, with the hope that the Kovacs effect could help identifying distributions of relaxation times, and/or provide some indirect evidence for a growing length scale in glassy systems.

\section{THE KOVACS EFFECT AND DOMAIN GROWTH}

The simplest out of equilibrium system is the one-dimensional Ising model with Glauber dynamics. This system does not order at any non zero temperature, but at sufficiently low temperatures the equilibrium domain size $\xi$ becomes large and for times shorter than the equilibration time, the dynamics is governed by the growth of the typical domain size as the square root of time. The energy, which is simply related to the average density of domain walls, plays in this model the rôle of the volume in Kovacs' experiments. When the system is prepared at $T_{1}$ for a time $t_{1}$ such that the average distance between the walls is equal to the equilibrium size at $T_{2}>T_{1}$, the out of equilibrium distribution of domain sizes at $T_{1}$ is more sharply peaked around its mean than the corresponding equilibrium distribution at $T_{2}$ -see Fig. 2. In particular, the number of small domains is depleted from its equilibrium value. Upon heating, the first effect is that some extra domain walls nucleate within the larger domains, causing the number of small domains 
(and the energy) to increase. The exact shape of Kovacs' hump can be computed in this model [17], and is found to be linear in time for small times, with a slope that increases with the temperature difference $T_{2}-T_{1}$, before reaching the (exponential) relaxation curve describing a simple quench from high temperatures. Note that the relaxation time is finite for all $T>0$ in this model; the rate of the final decay only depends on $T_{2}$, but not on $T_{1}$. As discussed by Brawer [17], this is qualitatively similar to the experimental curves reported in Fig. 1.

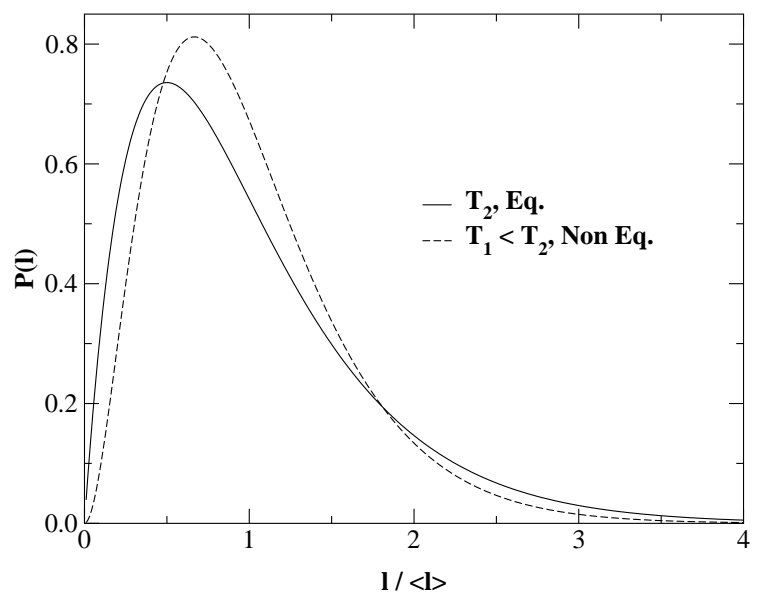

FIG. 2. Distribution of domain sizes in the one-dimensional Ising model corresponding to two different temperatures, such that the average domain size is identical in the two cases (from [17]). The out of equilibrium distribution $T_{1}<T_{2}$ (dotted line) is more sharply peaked than the corresponding equilibrium distribution at $T_{2}$ (plain line). Upon heating, small domains, initially less numerous, quickly appear within large ones.

In systems where the equilibrium domain size is infinite, or very large compared to the dynamical length corresponding to the experimental time scale, ${ }^{1}$ the mechanism for the Kovacs effect in coarsening systems is more generally the following [10,12]: after a time $t_{1}$ spent at $T_{1}$, the system orders up to a scale $\ell_{1}=\ell\left(t_{1}, T_{1}\right)$, leading to an excess energy (over the bulk contribution) due to the presence of domain walls with typical scale $\ell_{1}$. This excess energy density behaves as $\ell_{1}^{\Theta-d}$, where $\Theta$ is the exponent giving the scaling of the excess energy of a domain with its size (for example, $\Theta=d-1$ for the Ising model, and $\Theta=d-2$ in the XY-model). When the temperature is increased to $T_{2}$, the bulk energy density (within the domains) is suddenly too low compared to the equilibrium value at $T_{2}$. This bulk contribution to the energy density therefore increases rapidly by nucleating new domain walls within the large preexisting domains of size $\ell_{1}$. This picture was actually suggested in [14] to interpret an 'overshoot' effect in dipolar glasses which, with hindsight, is the precise counterpart of the Kovacs effect in these materials.

For larger times, the primary coarsening process resumes and the density of domain walls decreases, leading to a decrease of the total energy density. This decay is a priori expected to dominate when the length scale $\ell\left(t, T_{2}\right)$ associated to the dynamical processes initiated by the temperature change becomes of the order of $\ell_{1}$, i.e. after a time $\tau^{*}$ such that:

$$
\ell\left(\tau^{*}, T_{2}\right)=\ell_{1} .
$$

However, the time $\tau_{K}$ at which the maximum of the Kovacs hump occurs turns out to be, in general, much smaller than $\tau^{*}$ (but still much larger than the microscopic time scale $\tau_{0}$ ). More precisely, the following picture emerges from the exact computation of [10]:

- In the limit where $\ell\left(t, T_{2}\right)$ and $\ell_{1}$ are much larger than the lattice spacing $a$, the fast initial nucleation processes have taken place, and one can expect the energy density $E$ to take the following scaling form:

\footnotetext{
${ }^{1}$ If the equilibrium correlation length is small, then the Kovacs effect is trivial in the sense that only fast degrees of freedom need to reequilibrate. Only if the maximum of the Kovacs hump occurs at times much larger than the atomic time scales will one observe a non trivial effect when following the Kovacs protocol, where the contribution of fast degrees of freedom is removed by choosing $E\left(t_{1}^{+}\right)=E_{e q}\left(T_{2}\right)$. (See the discussion in the introduction).
} 


$$
\Delta E=E\left(t_{1}+t\right)-E_{e q}\left(T_{2}\right)=\Delta E_{K} \mathcal{F}\left(\frac{\ell\left(t, T_{2}\right)}{\ell_{1}}\right), \quad \ell\left(t, T_{2}\right) \gg a
$$

where $\Delta E_{K}$ is the height of the Kovacs' hump, and $\mathcal{F}(u) \sim u^{\Theta-d}$ when $u \rightarrow \infty$. Using the fact that $\Delta E$ should not depend on $t_{1}$ at large times, one finds $\Delta E_{K} \sim \ell_{1}^{\Theta-d}$, which means that the energy scale of the hump is of the order of the excess energy stored in the domain walls at $T_{1}$. As shown in [10], the above scaling form indeed holds exactly for the 2D XY model in the ordered critical phase, for which $\Theta=d-2$. One finds in that case $\mathcal{F}_{X Y}(u)=\left(1+u^{2}\right)^{-1}$.

- In the short time limit $\ell\left(t, T_{2}\right) \sim a$, one expects a nucleation contribution to $\Delta E$ responsible for the Kovacs hump. In the case of the critical XY model, where the thermal correlation length $\xi$ is infinite, one finds a power-law contribution [10]:

$$
\Delta E \approx \Delta E_{K}\left[1-\left(\frac{\ell\left(t, T_{2}\right)}{a}\right)^{\Theta-d}\right] .
$$

Note that this contribution vanishes for $t=0$, since $\ell\left(t=0, T_{2}\right)=a$, but cannot be written as a scaling function of $\ell / \ell_{1}$. This is at the origin of the difference between $\tau_{K}$ and $\tau^{*}$. The above results only hold if $\ell_{1} \ll \xi$. In the other limit where the correlation length $\xi$ is small, the above power-law is replaced by a fast exponential convergence. In this case $\tau_{K} \sim \tau_{0}$, and the Kovacs effect becomes trivial (it would actually disappear if the Kovacs protocol was used - see the previous footnote).

In this domain growth scenario, one finds the length scale $\ell_{1}$ (and therefore $\tau^{*}$ ) to be a decreasing function of $T_{2}-T_{1}$. Physically, this is because the bulk energy contribution is lower for smaller $T_{1}$; the residual domain wall energy density $\left(\sim \ell_{1}^{\Theta-d}\right)$ must then be larger in order to ensure that in the Kovacs protocol, the time $t_{1}$ is determined such that:

$$
E\left(t_{1}, T_{1}\right)=E_{e q}\left(T_{1}\right)+\ell_{1}^{\Theta-d}=E_{e q}\left(T_{2}\right) .
$$

For small $T_{2}-T_{1}$, one thus expects a linear relation $\Delta E_{K} \sim \ell_{1}^{\Theta-d} \propto C\left(T_{2}-T_{1}\right)$, where $C$ is the specific heat. Therefore, the qualitative dependence of both $\tau^{*}$ and $\Delta E_{K} \sim \ell_{1}^{\Theta-d}$ with $T_{2}-T_{1}$ is correctly predicted by this picture.

If the length $\ell(t, T)$ grows as a power of time, then from Eq. (3), $\Delta E / \Delta E_{K}$ is found to be a scaling function of $t / \tau^{*}$ in the limit of large times, where the initial (non scaling) contribution due to nucleation vanishes. Due to this non-scaling contribution, the scaling function $\mathcal{F}$ has a non zero value for small arguments: $\mathcal{F}\left(0^{+}\right)>0$. Therefore, in the domain growth scenario (including the equilibrium case discussed by Brawer and recalled above), the Kovacs hump does not rescale as a function of $t / \tau_{K}$, because the position of the maximum $\tau_{K}$ is determined by the non scaling nucleation contribution. The time should rather be rescaled by $\tau^{*}$ determined such that the amplitude of the hump has decreased by a factor two (say). By the same token, one expects to see systematic deviations from scaling in the regime $t \ll \tau^{*}$, due to the non scaling contribution of nucleation process.

We now turn to another soluble model that, interestingly, predicts a variety of shapes for the Kovacs hump, which in some regimes are very similar to the ones predicted by the domain growth model.

\section{THE KOVACS EFFECT IN THE TRAP MODEL}

\section{A. Definition of the model}

A simple model exhibiting glassy behaviour is the trap model, which has been extensively studied in the literature [18-20], and generalized to describe the rheology of soft glassy materials [21], or the dynamics of contacts in granular media [22]. In this model, a particle is trapped in potential wells, and can escape only through thermal activation. The depth (energy barrier) of the well is a random variable $E>0$ with an exponential a priori distribution $\rho(E)=$ $T_{g}^{-1} e^{-E / T_{g}}$. When the particle is in a trap $j$ of energy $E_{j}$, it will escape after a time $\Delta t$ distributed according to $p_{j}(\Delta t)=\tau_{j}^{-1} e^{-\Delta t / \tau_{j}}$, where $\tau_{j}=\tau_{0} e^{E_{j} / T}$ is the mean trapping time of the site $j$, and then chooses a new trap among all the others with a uniform probability. The microscopic time scale $\tau_{0}$ is taken as the time unit in the following. The energy scale $T_{g}$ turns out to be also the phase transition temperature. For $T>T_{g}$, the system equilibrates and behaves like a 'liquid', whereas for $T<T_{g}$, the lowest energy states become the most probable ones and the system 
never stops aging. Of course, this model should not be considered as a realistic microscopic model, but rather as a coarse-grained phase-space model -see the discussion in [23,24]. Also, an exponential distribution of energies might not be the most appropriate description of a given system. For example, recent simulations of Lennard-Jones systems [25] have shown that a Gaussian distribution of barriers is in fact more adequate. As noted in [20], the results of the exponential trap model can be extended to that case.

Due to its simplicity, this model allows one to obtain analytic expressions of many quantities of interest. As for coarsening models, we have chosen the energy as the natural observable that plays the rôle of the volume in Kovacs' experiments.

Let us now present the explicit calculation of the energy as a function of time, with the temperature protocol defined in the introduction. However, since fast degrees of freedom are absent in the trap model (there is no 'bottom of the wells' dynamics), one does not need to distinguish between $t_{1}^{-}$and $t_{1}^{+}$, as is important both experimentally and in models with microscopic degrees of freedom (see above for a discussion of this point). Two different cases have been considered in details. In the first one, the temperatures $T_{1}$ and $T_{2}$ are both above $T_{g}$, but close to it, so that the system eventually equilibrates, but with very long relaxation times. In the second case, both temperatures are below $T_{g}$, so that the system is in the aging regime where equilibration is never achieved. Finally, we only briefly discuss the 'mixed' case where $T_{1}<T_{g}<T_{2}$. The original Kovacs experiment corresponds to the first case, since the volume is seen to relax towards its equilibrium value at $T_{2}$, used as the reference energy. In the second case, the time $t_{1}$ at which temperature is shifted is in fact arbitrary, but interesting scaling properties appear.

\section{B. Case $T>T_{g}$ : relaxation towards equilibrium}

We shall use a continuous energy description (see [20]), i.e. the system is described by the probability $P_{\mathrm{T}}(E, t)$ to be in a state with an energy (barrier) $E$ at time $t$ and temperature $T$, which evolves according to the following Master equation:

$$
\frac{\partial P_{\mathrm{T}}}{\partial t}(E, t)=-e^{-E / T} P_{\mathrm{T}}(E, t)+\omega(t) \rho(E)
$$

with $\omega(t)=\int_{0}^{\infty} d E^{\prime} e^{-E^{\prime} / T} P_{\mathrm{T}}\left(E^{\prime}, t\right)$ is the average hopping rate. For $T>T_{g}, P_{\mathrm{T}}(E, t)$ relaxes towards the equilibrium distribution $P_{\mathrm{T}}^{e q}(E)=Z^{-1} e^{E / T}$. So the interesting quantity to study is the deviation from equilibrium, i.e. the distribution $p_{\mathrm{T}}(E, t)$ defined as $p_{\mathrm{T}}(E, t)=P_{\mathrm{T}}(E, t)-P_{\mathrm{T}}^{e q}(E)$. Let us first focus on a simple isothermal quench from a given initial condition $P_{0}(E)=P_{\mathrm{T}_{0}}^{e q}(E)$. The evolution of $p_{\mathrm{T}}(E, t)$ can be computed using a time Laplace transform, and if $T_{0}>T$, the asymptotic behaviour of the distribution becomes independent of the initial condition $P_{0}(E)$, yielding:

$$
\hat{p}(E, s)=\frac{\left(\beta_{g}-\beta\right) e^{-\left(\beta_{g}-\beta\right) E}}{1+s e^{\beta E}}\left[\Gamma(\theta) \Gamma(2-\theta) s^{\theta-2}-e^{\beta E}\right]
$$

where $\beta=1 / T$ and $\theta=T / T_{g}$ is the reduced temperature. Let us define the energy deviation $\varepsilon_{\mathrm{T}}(t)=\left|E_{\mathrm{T}}(t)-E_{\mathrm{T}}^{e q}\right|$. Note that in the following, energies are understood to be true physical energies, i.e. the opposite of the energy barriers: $\varepsilon_{\mathrm{T}}(t)=-\int_{0}^{\infty} d E E p_{\mathrm{T}}(E, t)$. This last quantity can be computed from $\hat{p}(E, s)$, which gives:

$$
\varepsilon_{\mathrm{T}}(t)=\frac{T}{t^{\theta-1}}\left[\Gamma(\theta) \ln t-\Gamma^{\prime}(\theta)\right]
$$

Hence, the energy relaxation above $T_{g}$ is (up to a logarithmic correction) a power law with an exponent that becomes small for $T \rightarrow T_{g}$. The time $t_{1}$ when the temperature has to be raised from $T_{1}$ to $T_{2}$ in the Kovacs procedure is defined by $E_{\mathrm{T}_{1}}\left(t_{1}\right)=E_{\mathrm{T}_{2}}^{e q}$, or equivalently $\varepsilon_{\mathrm{T}_{1}}\left(t_{0}\right)=E_{\mathrm{T}_{2}}^{e q}-E_{\mathrm{T}_{1}}^{e q}$. Thus $t_{1}$ is determined by the equation:

$$
\frac{1}{t_{1}^{\theta_{1}-1}}\left[\Gamma\left(\theta_{1}\right) \ln t_{1}-\Gamma^{\prime}\left(\theta_{1}\right)\right] \approx \frac{\theta_{2}-\theta_{1}}{\left(\theta_{1}-1\right)^{2}}
$$

Note that in order to be consistent, the above equation assumes that $\theta_{2}-\theta_{1} \ll\left(\theta_{1}-1\right)^{2} \ll 1$, in which case $t_{1} \gg \tau_{0}(=1)$.

Now using the distribution $p_{\mathrm{T}_{2}}\left(E, t_{0}\right)=p_{\mathrm{T}_{1}}\left(E, t_{0}\right)+P_{\mathrm{T}_{1}}^{e q}(E)-P_{\mathrm{T}_{2}}^{e q}(E)$ as initial condition in the Master equation, one can compute the further evolution of the energy at $T_{2}$ at time $t_{1}+t$. A time scale $\tau^{*}=t_{1}^{\gamma}$ naturally appears (with $\left.\gamma=\theta_{1} / \theta_{2}\right)$. Defining the energy variation $\Delta E(t)=E\left(t_{1}+t\right)-E\left(t_{1}\right)$, one finds in the short-time regime $1 \ll t \ll \tau^{*}$ : 


$$
\Delta E(t) \approx \frac{T_{1}}{t_{1}^{\theta_{1}-1}}\left[\ln t_{1}+\frac{1}{\theta_{1}-1}\right]-\left(1+\frac{1}{t_{1}^{\theta_{1}-1}}\right) \frac{T_{2}}{t^{\theta_{2}-1 / \gamma}}\left[\ln t+\gamma_{E}\right]+\frac{T_{2}}{t^{\theta_{2}-1}}\left[\ln t+\gamma_{E}\right]
$$

where $\gamma_{E}=-\Gamma^{\prime}(1)$ is the Euler constant. Interestingly, this behaviour is very similar to that found for the coarsening process. Indeed, in the limit $\theta_{2}-\theta_{1} \ll\left(\theta_{1}-1\right)^{2}$, the two power laws in the previous equation, $\theta_{2}-1 / \gamma$ and $\theta_{2}-1$ are very close to each other, and the expression can be simplified as:

$$
\Delta E(t) \approx \frac{T_{1}}{t_{1}^{\theta_{1}-1}}\left(\left[\ln t_{1}+\frac{1}{\theta_{1}-1}\right]-\frac{1}{t^{\theta_{2}-1}}\left[\ln t+\gamma_{E}\right]\right) .
$$

Therefore, the maximum of the Kovacs hump is given (in the considered limit) by:

$$
\Delta E_{K} \approx \frac{T_{1}}{t_{1}^{\theta_{1}-1}} \ln t_{1} \approx \frac{\theta_{2}-\theta_{1}}{\left(\theta_{1}-1\right)^{2}} .
$$

The approach to this maximum is described by a power law of time with a logarithmic correction. This is not very different from the coarsening model discussed in the previous section. Note that the height of the hump is again linear in $T_{2}-T_{1}$ for small temperature differences, as was the case for domain growth. Note also that $\tau^{*}=t_{1}^{\gamma}$ is a decreasing function of $T_{2}-T_{1}$, in agreement with experimental results.

In the long time regime $t \gg \tau^{*}$, one recovers as expected the isothermal quench result Eq. (8) at temperature $T_{2}$ :

$$
\Delta E(t)=\frac{T_{2}}{t^{\theta_{2}-1}}\left[\Gamma\left(\theta_{2}\right) \ln t-\Gamma^{\prime}\left(\theta_{2}\right)\right]
$$

This late time result can again be put in a scaling form (up to logarithmic corrections):

$$
\Delta E(t)=\Delta E_{K} \mathcal{G}\left(\frac{t}{\tau^{*}}\right) \quad \tau^{*}=t_{1}^{\gamma}
$$

but the early time regime Eq. (11) fails to scale. Only when $T_{1}, T_{2} \rightarrow T_{g}$, does one find that the maximum time $\tau_{K}$ coincides with $\tau^{*}$. More generally, and as for domain growth, one has $\tau_{K} \ll \tau^{*}$.

\section{The aging case $\left(T_{1}, T_{2}<T_{g}\right)$}

We now turn to the aging case where the shape of the hump is found to be qualitatively different. We consider the case where both $T_{1}$ and $T_{2}$ are less than $T_{g}$. In this case, the system never converges to an equilibrium state, but keeps on aging, so that the situation is different from that of the Kovacs original experiment, but could in principle also be investigated experimentally. Since the equilibrium energy at $T_{2}$ does not exist, we choose to shift the temperature from $T_{1}$ (initially reached at $t=0$ ) to $T_{2}$ after a waiting time $t_{w}$ (which plays the role of $t_{1}$ in the previous sections).

\section{Probability distribution and Green function}

The continuous energy Master equation (6) does not admit anymore a stationary solution. The resulting dynamical distribution can be computed using the Laplace transform $\hat{P}_{\mathrm{T}}\left(E, s_{w}\right)$ (in the time domain) of $P_{\mathrm{T}}\left(E, t_{w}\right)$, where $t_{w}$ is the waiting time since the quench from high temperatures. One finds, in the asymptotic regime $s_{w} \rightarrow 0$ (or $t_{w} \rightarrow \infty$ ):

$$
\hat{P}_{\mathrm{T}}\left(E, s_{w}\right) \simeq \hat{\Pi}_{\mathrm{T}}(E, s) \equiv \frac{\sin \pi \theta}{\pi} \frac{\beta e^{\beta E}}{\left(1+s e^{\beta E}\right)\left(s e^{\beta E}\right)^{\theta}},
$$

where $\theta \equiv T / T_{g}$. Since $s \hat{\Pi}_{\mathrm{T}}(E, s)$ is a function of the product $s e^{\beta E}, \Pi_{\mathrm{T}}(E, t)$ depends only on the scaling variable $\xi=e^{\beta E} / t$. Then one can turn to the computation of the energy variation after the temperature shift. 


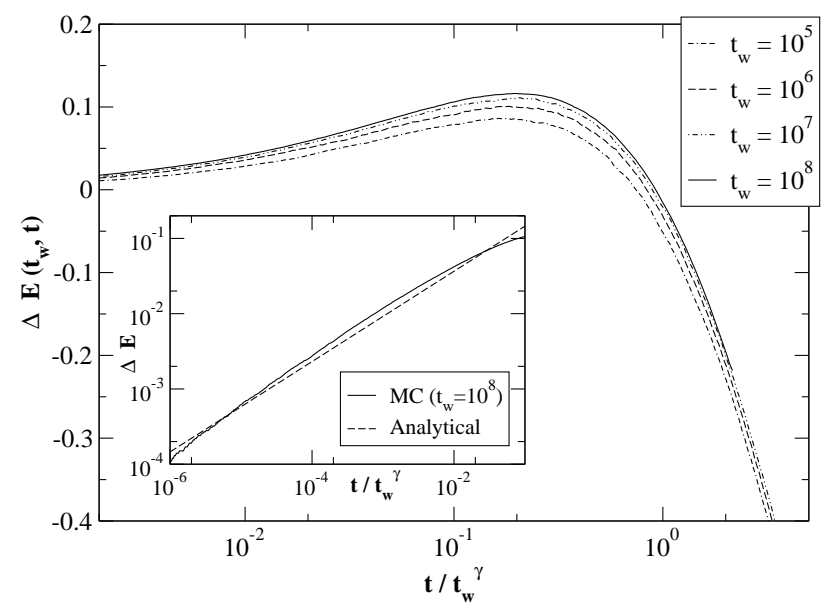

FIG. 3. Plot of $\Delta E\left(t_{w}, t\right)$ in the trap model as a function of the scaling variable $t / t_{w}^{\gamma}$ for $t_{w}=10^{5}, 10^{6}, 10^{7}$ and $10^{8}(M o n t e$ Carlo data), $\theta_{1}=0.5$ and $\theta_{2}=0.6$. Inset: comparison between Monte Carlo data $\left(t_{w}=10^{8}\right)$ and the analytical prediction of the short time behaviour -see Eq. (21)-for the same temperatures as above.

\section{Computation of the energy variation}

The detailed calculation of the energy variation $\Delta E\left(t_{w}, t\right)$ between time $t_{w}$ (when temperature is shifted from $T_{1}$ to $T_{2}$ ) and $t_{w}+t$ is given in Appendix A. Here we shall only summarize the main steps of the calculation, and emphasize physical interpretations and conclusions. From a technical point of view, a useful tool in order to compute $\Delta E\left(t_{w}, t\right)$ is the Green function $G_{\mathrm{T}}\left(E, E_{0}, t\right)$ defined as the probability to have the energy $E$ at time $t_{w}+t$ given that the energy was $E_{0}$ at time $t_{w}$. This Green function is computed, as for $P_{\mathrm{T}}\left(E, t_{w}\right)$, using a Laplace transform, with $s$ the Laplace variable. One finds, for $s \tau_{0} \ll 1\left(t \gg \tau_{0}\right)$, the following asymptotic expression:

$$
\hat{G}_{\mathrm{T}}\left(E, E_{0}, s\right)=\frac{e^{\beta E_{0}}}{1+s e^{\beta E_{0}}} \delta\left(E-E_{0}\right)+\frac{1}{1+s e^{\beta E_{0}}} \hat{\Pi}_{\mathrm{T}}(E, s)
$$

Thanks to the Markovian properties of the dynamics, the Green function does not depend on $t_{w}$, but only on the time difference $t$. One can express the average energy $\bar{E}\left(t_{w}+t\right)$ at time $t_{w}+t$ using the Green function:

$$
\bar{E}\left(t_{w}+t\right)=-\int_{0}^{\infty} d E \int_{0}^{\infty} d E_{0} E G_{\mathrm{T}_{1}}\left(E, E_{0}, t\right) P_{\mathrm{T}_{2}}\left(E_{0}, t_{w}\right)
$$

where the minus sign accounts for the fact that the variable $E$ (i.e. the energy barrier) is actually the opposite of the true energy. The energy variation $\Delta E\left(t_{w}, t\right)$ is then:

$$
\begin{aligned}
\Delta E\left(t_{w}, t\right) & \equiv \bar{E}\left(t_{w}+t\right)-\bar{E}\left(t_{w}\right) \\
& =-\int_{0}^{\infty} d E \int_{0}^{\infty} d E_{0}\left(E-E_{0}\right) G_{\mathrm{T}_{1}}\left(E, E_{0}, t\right) P_{\mathrm{T}_{2}}\left(E_{0}, t_{w}\right)
\end{aligned}
$$

After a few calculations (see Appendix A), one can show that $\Delta E\left(t_{w}, t\right)$ exhibits a kind of 'sub-aging' scaling (see also [26]):

$$
\Delta E\left(t_{w}, t\right)=\psi\left(\frac{t}{t_{w}^{\gamma}}\right)
$$

where $\gamma=T_{1} / T_{2}<1$. One sees that the energy evolves on a typical time scale given by $\tau_{K}=\tau^{*}=t_{w}^{\gamma}$, which is expected from a simple activation argument. One can also study the asymptotic (short time and late time) behaviour of this scaling function. Concerning the short time behaviour, it is necessary to distinguish between two cases.

- If $\gamma>1-\theta_{1}$ (small temperature shifts) then $\Delta E\left(t_{w}, t\right)$ is found to be singular at short times:

$$
\Delta E\left(t_{w}, t\right) \simeq K_{>}\left(\frac{t}{t_{w}^{\gamma}}\right)^{\left(1-\theta_{1}\right) / \gamma} \quad t \ll t_{w}^{\gamma}
$$


- If on the contrary $\gamma<1-\theta_{1}$, one finds a linear $t$ dependence in the short time regime (with logarithmic corrections):

$$
\Delta E\left(t_{w}, t\right) \simeq K_{<}\left(\ln \frac{t_{w}^{\gamma}}{t}+C\right) \frac{t}{t_{w}^{\gamma}} \quad t \ll t_{w}^{\gamma}
$$

The coefficients $K_{>}, K_{<}$and $C$ appearing in Eqs. (21, 22) are given in Appendix A -see Eqs. $(64,70,71)-$ and are found to be positive for $\theta_{1}<\theta_{2}$. Therefore $\Delta E\left(t_{w}, t\right)$ is positive for short times, and the Kovacs effect has the expected sign.

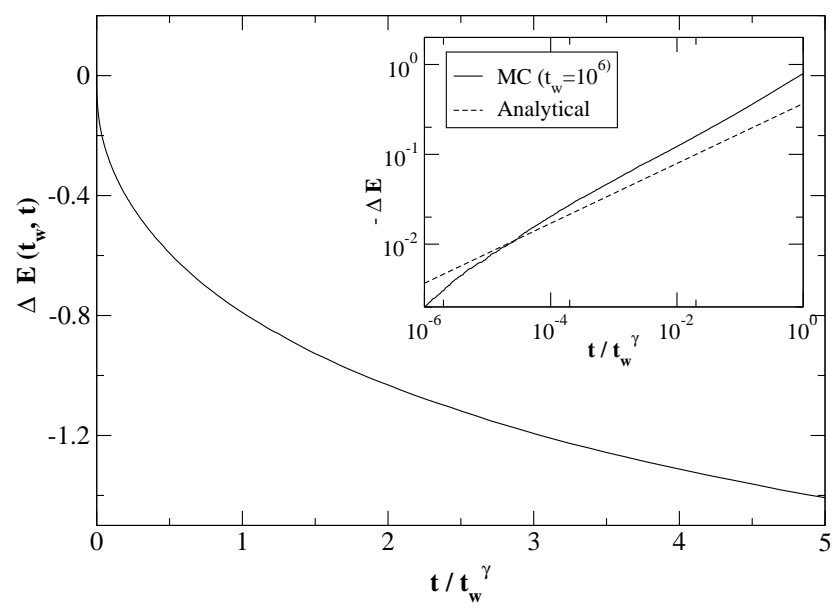

FIG. 4. Plot of $\Delta E\left(t_{w}, t\right)$ in the trap model as a function of the scaling variable $t / t_{w}^{\gamma}$ for $t_{w}=10^{6}$ (Monte Carlo data), in the case of a negative temperature shift: $\theta_{1}=0.6$ and $\theta_{2}=0.5$. Inset: comparison between Monte Carlo data and the analytical prediction of the short time behaviour -see Eq. (21); note that finite size effects are strong in this case.

Note also that the coefficient $K_{>}$vanishes linearly when $\theta_{1} \rightarrow \theta_{2}$. This is expected: if no temperature jump occurs, the energy variation should be regular, i.e. linear in $t$. Moreover, if $\theta_{1}>\theta_{2}$ (negative temperature shift, $\gamma>1$ ), the above calculation is still valid, with a negative $K_{>}$, and a non trivial exponent $\left(1-\theta_{1}\right) / \gamma$. The Kovacs hump becomes in this case a Kovacs trough. Monte-Carlo data are compared with these analytical predictions in Figs. 3 and 4 , showing a rather good agreement. Note that the scaling Eq. (20) is only approximate for finite $t_{w}$. A better rescaling can be obtained in the case $\theta_{1}<\theta_{2}$ by plotting $\Delta E\left(t, t_{w}\right) / \Delta E_{K}$ as a function of $t / \tau_{K}$, where $\Delta E_{K}$ is the maximum value of $\Delta E\left(t_{w}, t\right)$, reached at $t=\tau_{K} \simeq t_{w}^{\gamma}$. Eq. (20) means that asymptotically, $\Delta E_{K}$ becomes independent of $t_{w}$.

Finally, the long time behaviour is easy to analyze: one can show that $P_{\mathrm{T}}\left(E, t_{w}+t\right)$ behaves asymptotically in the same way whatever the initial condition $P_{\mathrm{T}}\left(E, t_{w}\right)$. The system behaves, at late time, as if it had been quenched directly from high temperature (see Fig. 5). This means that in this limit $\bar{E}\left(t_{w}+t\right)$ does not depend on $t_{w}$, but only on $t$ :

$$
\bar{E}\left(t_{w}+t\right) \simeq \bar{E}_{\text {late }}(t) \equiv T_{2}\left[\Gamma^{\prime}(1)-\pi \cot \pi \theta_{2}\right]-T_{2} \ln t \quad t \gg t_{w}^{\gamma}
$$

where $\bar{E}_{\text {late }}(t)$ is the average energy at a (large) time $t$ after a quench from a high temperature. So $\Delta E\left(t_{w}, t\right)$ is simply given by:

$$
\Delta E\left(t_{w}, t\right)=\bar{E}_{\text {late }}(t)-\bar{E}\left(t_{w}\right) \quad t \gg t_{w}^{\gamma}
$$




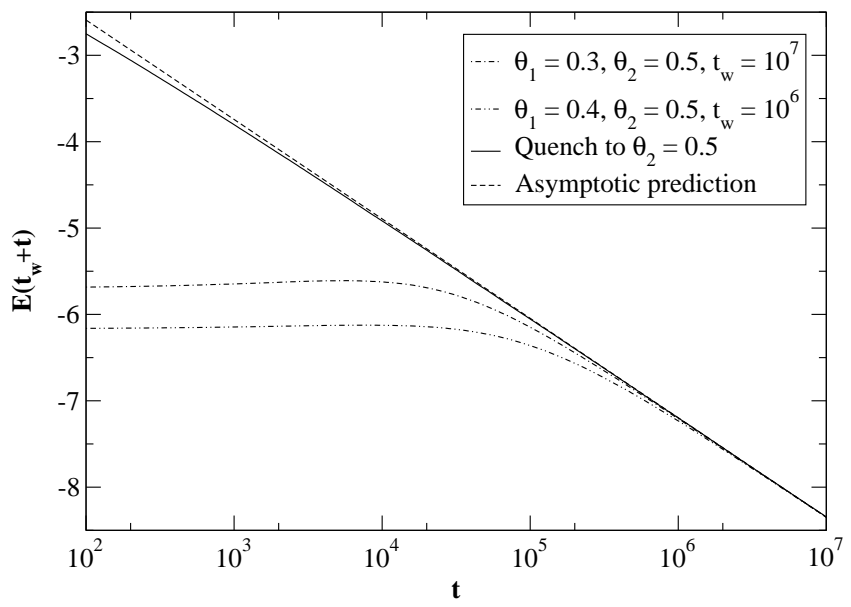

FIG. 5. Comparison of the late time behaviour of $E\left(t_{w}+t\right)=\Delta E\left(t_{w}, t\right)+E\left(t_{w}\right)$ in the trap model as a function of $t$, for temperatures $\theta_{1}=0.3$ and $0.4, \theta_{2}=0.5$ and waiting times $t_{w}=10^{7}$ and $10^{6}$ respectively (dot-dash) with a direct quench from infinite temperature to $\theta_{2}=0.5\left(t_{w}=0\right.$, full line). The asymptotic analytical prediction is also shown (dashed line).

\section{The mixed case $\left(T_{1}<T_{g}<T_{2}\right)$}

For completeness, and since this is also an interesting situation, we briefly mention the results obtained in the trap model in the case where the glass transition temperature $T_{g}$ lies between $T_{1}$ and $T_{2}: T_{1}<T_{g}<T_{2}$. This case is worth studying, since the system can eventually equilibrate at the final temperature $T_{2}$, with long relaxation times (assuming $T_{2}$ is close to $T_{g}$ ), but $T_{1}$ can be varied in the whole range $0<T_{1}<T_{g}$ and not only in the vicinity of $T_{g}$. Interestingly, one finds the same short time singularities as in the aging case $\left(T_{1}<T_{2}<T_{g}\right)$ studied above :

$$
\begin{aligned}
& \Delta E\left(t_{1}, t\right) \sim\left(\frac{t}{t_{1}^{\gamma}}\right)^{\left(1-\theta_{1}\right) / \gamma} \quad 1-\theta_{1}<\gamma \\
& \Delta E\left(t_{1}, t\right) \sim \frac{t}{t_{1}^{\gamma}} \quad 1-\theta_{1}>\gamma
\end{aligned}
$$

with however prefactors and logarithmic corrections which are different from that found in the aging case. In the long time regime, one naturally finds a convergence of $\Delta E\left(t, t_{1}\right)$ proportionnal to $t^{-\left(\theta_{2}-1\right)}$, as in the case $T_{g}<T_{1}<T_{2}$. As a result, one sees as could have been expected that the short time regime is generically dominated by the thermal history before the temperature shift, whereas the long time behaviour depends only on the final temperature $T_{2}$.

\section{E. Discussion - 'Fronts' in the energy distribution}

It is interesting to discuss how the distribution of energies $P(E, t)$ evolves when the temperature is shifted from $T_{1}$ to $T_{2}>T_{1}$. This is illustrated in Fig. 6: at the lowest temperature, the probability of small (negative) energies is depressed. When the temperature is raised, the system obviously re-equilibrates fastest in the region of small energies since this corresponds to the smallest relaxation times. The probability 'hole' is thus rapidly filled, leading to an increase of the average energy, and thus to the Kovacs effect. As time increases, the equilibration progresses as a kind of 'front' in energy space, as shown in Fig. 6. Only at later times does the peak of the distribution move to larger (negative) energies. It is interesting to realize that this picture is in fact very close the one emerging from the coarsening model where short scales re-equilibrate fast and lead to an increase of the average energy, before larger length scales resume the coarsening process (see Fig. 2, and the discussion of Section II).

The conclusion from the 'domain growth' interpretation of the Kovacs effect presented in the previous section is that a quantitative analysis of the Kovacs effect might give one a unique tool to investigate experimentally the problem of growing length scales or the statistics of trapping times in glassy systems, a topic of huge current interest [27-30,25,31,32]. However, as demonstrated above, the quantitative predictions of the trap model are in fact very similar to that of domain growth. As discussed recently in [33], the physical difference between the two pictures is not as obvious as it might first seem. In particular, the trap model description implicitly assumes the existence of an 
underlying 'coherence length' [23]; conversely, domain growth models may naturally generate a non trivial distribution of relaxation times [33]. A possible discrimination might lie in the temperature dependence of the short-time and long-time exponents that describe the Kovacs hump. While a temperature dependence is expected in an activated trap like description, it is less natural for power-law domain growth. On the other hand, more complicated (logarithmic) growth laws can mimic power-laws with a temperature dependent exponent $[34,12]$.

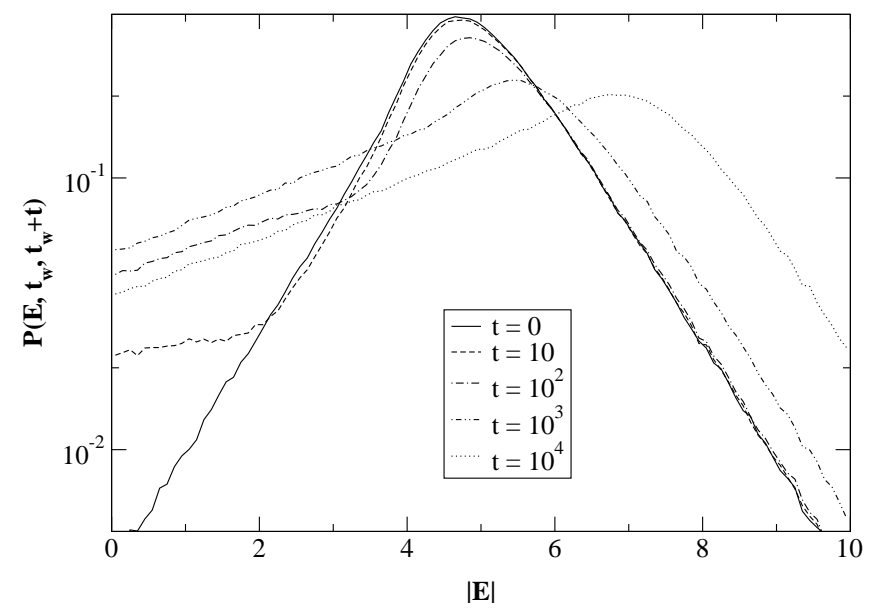

FIG. 6. Dynamical energy distribution $P\left(E, t_{w}, t_{w}+t\right)$ at time $t_{w}+t$ after a (positive) temperature shift at $t_{w}$, with $t_{w}=10^{4}$, $\theta_{1}=0.5$ and $\theta_{2}=0.8$ (trap model). Time $t$ ranges from $t=0$ to $t=t_{w}$. One clearly sees the propagation of a 'front' at small energies, associated to the re-equilibration of the short time scales, before the global drift of the distribution starts again.

\section{SUMMARY AND CONCLUSIONS}

Although the Kovacs effect has been known for fourty years, its quantitative interpretation has not been much developed until recently. In view of the fact that this effect is generic and observed in a variety of different 'glassy' systems and models (such as the ones studied in the present paper), it is important to establish which type of microscopic information one can extract from the quantitative analysis of the 'Kovacs hump'. Qualitatively, the Kovacs effect reflects the heterogeneity of the system: fixing the overall (macroscopic) value of the volume or energy does not prevent the existence of local fluctuations, which keep track of the system history. A more complete description of the system therefore requires to deal with full distribution functions, and not only with averages. In the two models studied in this paper, this distribution function is that of domain sizes in domain growth models, and that of relaxation times in the trap model. These models lead to precise, quantitative predictions for the shape of the Kovacs hump, which are summarized in Table I, and by the following phenomenological equation that describes the Kovacs hump [inspired from both domain growth and trap models -see Eqs. $(3,11,13)]$ :

$$
\Delta E=\Delta E_{K}\left[\frac{\tau^{* \nu}}{\left(t+\tau^{*}\right)^{\nu}}-\frac{\varphi}{t^{\nu}}\right]
$$

In domain growth models, the exponent $\nu$ is equal to $(d-\Theta) / z$, where $z$ is the dynamical exponent relating length and time: $\ell \sim t^{1 / z}$. The first term corresponds to the long time contribution of already grown domains, and the second term to the excess energy created by the transient nucleation of new domains. A similar interpretation of the two terms can be given within the trap model, with $\nu=T / T_{g}-1$ (for an exponential distribution of barriers). Note that from Eq. (27), one sees that the data should re-scale as $\Delta E_{K} \mathcal{G}\left(\frac{t}{\tau^{*}}\right)$ when $\tau^{*}$ becomes large. The position of the maximum $\tau_{K}$ extracted from Eq. (27) is, in the limit $\tau^{*} \gg 1$, given by:

$$
\tau_{K} \approx\left(\varphi \tau^{*}\right)^{\frac{1}{\nu+1}} \ll \tau^{*}
$$

so that the correct scaling variable is not $t / \tau_{K}$, except in the limit $\nu \rightarrow 0$, where $\tau_{K}$ and $\tau^{*}$ coincide.

We hope that these results will motivate new, systematic experiments. It would in particular be valuable to test Eq. (27). It would be very interesting to extract from a detailed analysis of the Kovacs effect a quantitative determination of the distribution of relaxation times in glassy systems, and its temperature dependence. This distribution could 
then be compared with other direct, dynamical determinations. Another situation worth investigating experimentally, suggested by the present study, is the out of equilibrium (aging) Kovacs effect, where both temperatures are kept well below the glass temperature $T_{g}$.

Finally, from a theoretical point of view, it would be worth studying the predictions of the mean field (p-spin) spin-glass for the shape of the Kovacs hump. As is well known (see e.g. [35]), the dynamical equations for this system are identical to the Mode-Coupling equations for structural glasses. Although we expect on general grounds that the results of this model should be again quite similar to those obtained in the present study, it would be interesting to check this assertion in more details.

\begin{tabular}{|c|c|c|c|}
\hline \hline & Domain growth $\left(\ell_{e q}=\infty\right)$ & Trap model $T_{1}>T_{g}$ & Trap model $T_{1}<T_{g}$ \\
\hline Preparation time $t_{1}$ & $\ell\left(t_{1}, T_{1}\right)^{\Theta-d} \propto T_{2}-T_{1}$ & $t_{1}^{1-\theta_{1}} \ln t_{1} \propto T_{2}-T_{1}$ & $t_{w}$ \\
\hline Height of the hump $\Delta E_{K}$ & $T_{2}-T_{1}$ & $T_{2}-T_{1}$ & $T_{2}-T_{1}$ \\
\hline Characteristic time $\tau^{*}$ & $\ell\left(\tau^{*}, T_{2}\right)=\ell\left(t_{1}, T_{1}\right)$ & $\tau^{*}=t_{1}^{T_{1} / T_{2}}$ & $\tau^{*}=t_{w}^{T_{1} / T_{2}}$ \\
\hline Hump time $\tau_{K}$ & $\tau_{K} \sim\left(\tau^{*}\right)^{\frac{1}{\nu+1} \ll \tau^{*}}$ & $\tau_{K} \sim\left(\tau^{*}\right)^{\frac{1}{\nu+1} \ll \tau^{*}}$ & $\tau_{K} \sim \tau^{*}$ \\
\hline$\Delta E$ at early time & $\Delta E_{K}\left(1-\ell^{\Theta-d}\right)$ & $\Delta E_{K}\left(1-t^{-\nu} \ln t\right)$ & $\Delta E_{K}\left(t / \tau^{*}\right)^{\left(1-\theta_{1}\right) / \gamma}$ \\
\hline$\Delta E$ at late time & $\Delta E_{K}\left(\ell_{1} / \ell\right)^{d-\Theta}$ & $\Delta E_{K}\left(\tau^{*} / t\right)^{\nu}$ & $-T_{2} \ln \left(t / t_{w}^{\gamma}\right)$ \\
\hline Exponent $\nu$ & $\nu=(d-\Theta) / z$ & $\nu=\theta_{2}-1$ & \\
\hline \hline
\end{tabular}

TABLE I. Summary of the different results and regimes for the Kovacs hump, in the limit where $T_{1} \rightarrow T_{2}^{-}$. We denote by $t_{1}$ or $t_{w}$ the time spent at the lowest temperature $T_{1}, \Theta$ and $z$ the energy and dynamical exponents for domain growth, $\gamma$ the ratio $\gamma=T_{1} / T_{2}$ and $\theta_{1,2}$ the reduced temperatures $\theta_{1,2}=T_{1,2} / T_{g}$. 


\section{ACKNOWLEDGMENTS}

We want to thank E. Pitard and M. Sasaki (who both took part in an early stage of this work) and L. Berthier, L. Cugliandolo, J. Kurchan and E. Vincent for very useful discussions. We also thank a referee for pointing out a conceptual error in the Lennard-Jones simulation results presented in the first version of this manuscript.

\section{APPENDIX: DETAILED CALCULATION IN THE TRAP MODEL}

\section{A. Probability distribution and Green function}

The Master equation of the trap model reads:

$$
\frac{\partial P_{\mathrm{T}}}{\partial t}(E, t)=-e^{-\beta E} P_{\mathrm{T}}(E)+\omega(t) \rho(E)
$$

with $\omega(t)=\int_{0}^{\infty} d E^{\prime} e^{-\beta E^{\prime}} P_{\mathrm{T}}\left(E^{\prime}, t\right)$, and $\beta=\frac{1}{T}$. $E$ is a positive variable, the depth of the traps, and is actually the opposite of the true energy of the states. This Master equation has to be supplemented by an initial condition:

$$
P_{\mathrm{T}}(E, t=0)=P_{0}(E)
$$

where $P_{0}(E)$ is a given (arbitrary) probability distribution. We also take an exponential density of states, $\rho(E)=$ $T_{g}^{-1} e^{-E / T_{g}}$. Introducing the Laplace transform $\hat{P}_{T}(E, s)$ with respect to $t$ defined as:

$$
\hat{P}_{\mathrm{T}}(E, s)=\int_{0}^{\infty} d t e^{-s t} P_{\mathrm{T}}(E, t)
$$

the Master equation becomes:

$$
s \hat{P}_{\mathrm{T}}(E, s)-P_{0}(E)=-e^{-\beta E} \hat{P}_{\mathrm{T}}(E, s)+\hat{\omega}(s) \rho(E)
$$

Solving for $\hat{P}_{\mathrm{T}}(E, s)$, one has:

$$
\hat{P}_{\mathrm{T}}(E, s)=\frac{P_{0}(E)}{s+e^{-\beta E}}+\frac{\hat{\omega}(s) \rho(E)}{s+e^{-\beta E}}
$$

$\hat{\omega}(s)$ is determined by multiplying Eq. (33) by $e^{-\beta E}$ and integrating over $E$. The distribution $\hat{P}_{\mathrm{T}}(E, s)$ is then given by:

$$
\hat{P}_{\mathrm{T}}(E, s)=\frac{e^{\beta E}}{1+s e^{\beta E}} P_{0}(E)+\frac{1}{s} \frac{e^{\beta E} \rho(E)}{1+s e^{\beta E}} \hat{\varphi}(s)\left[\int_{0}^{\infty} d E \frac{e^{\beta E} \rho(E)}{1+s e^{\beta E}}\right]^{-1}
$$

with $\hat{\varphi}(s)$ defined as:

$$
\hat{\varphi}(s)=\int_{0}^{\infty} d E \frac{P_{0}(E)}{1+s e^{\beta E}}
$$

Integrating Eq. (34) over $E$ allows to check that $\hat{P}_{\mathrm{T}}(E, s)$ is well normalized, i.e. $\int_{0}^{\infty} d E \hat{P}_{\mathrm{T}}(E, s)=1 / s$. In order to compute the variation of the energy after a temperature shift, one has to introduce the Green function $G_{\mathrm{T}}\left(E, E_{0}, t\right)$ defined as the probability for the system to have energy $E$ at time $t_{w}+t$ given that the energy was $E_{0}$ at time $t_{w}$, if the bath temperature is $T$. Note that since the process is Markovian, the Green function depends only on the time difference $t$, and not on $t_{w}$. The Green function in Laplace space $\hat{G}_{\mathrm{T}}\left(E, E_{0}, s\right)$ is straightforwardly obtained from Eq. (34) choosing $P_{0}(E)=\delta\left(E-E_{0}\right)$ :

$$
\hat{G}_{\mathrm{T}}\left(E, E_{0}, s\right)=\frac{e^{\beta E_{0}}}{1+s e^{\beta E_{0}}} \delta\left(E-E_{0}\right)+\frac{1}{s} \frac{1}{1+s e^{\beta E_{0}}} \frac{e^{\beta E} \rho(E)}{1+s e^{\beta E}}\left[\int_{0}^{\infty} d E \frac{e^{\beta E} \rho(E)}{1+s e^{\beta E}}\right]^{-1}
$$

As shown in [20], the energy distribution $P_{\mathrm{T}}(E, t)$ takes a scaling form for large times. Indeed, from Eq. (34), one has for $s \rightarrow 0$ : 


$$
\hat{P}_{\mathrm{T}}(E, s)=\frac{1}{s} \frac{e^{\beta E} \rho(E)}{1+s e^{\beta E}}\left[\int_{0}^{\infty} d E \frac{e^{\beta E} \rho(E)}{1+s e^{\beta E}}\right]^{-1}=\frac{\sin \pi \theta}{\pi} \frac{\beta e^{\beta E}}{\left(1+s e^{\beta E}\right)\left(s e^{\beta E}\right)^{\theta}} \equiv \hat{\Pi}_{\mathrm{T}}(E, s)
$$

which defines the asymptotic distribution $\hat{\Pi}_{\mathrm{T}}(E, s)$. The reduced temperature $\theta=T / T_{g}$ has also been introduced. Its inverse Laplace transform $\Pi_{\mathrm{T}}(E, t)$ satisfies a scaling relation in the variable $\xi=\frac{e^{E / T}}{t}$ :

$$
\Pi_{\mathrm{T}}(E, t)=\beta \xi g(\xi)
$$

One finds $g(\xi)$ by inverting the Laplace transform given by Eq. (37):

$$
g(\xi)=\frac{\sin \pi \theta}{\pi \Gamma(\theta)} \frac{1}{\xi} e^{-1 / \xi} \int_{0}^{1 / \xi} d u u^{\theta-1} e^{u}
$$

So for large times, the Green function is given by:

$$
\hat{G}_{\mathrm{T}}\left(E, E_{0}, s\right)=\frac{e^{\beta E_{0}}}{1+s e^{\beta E_{0}}} \delta\left(E-E_{0}\right)+\frac{1}{1+s e^{\beta E_{0}}} \hat{\Pi}_{\mathrm{T}}(E, s)
$$

We now consider the following thermal history: at the initial time, the system is quenched from $T_{0}>T_{g}$ to $T_{1}<T_{g}$; at time $t_{w}$, it is re-heated to a temperature $T_{2}$ satisfying $T_{1}<T_{2}<T_{g}$. One is interested in the subsequent evolution of the energy, at time $t_{w}+t$. The probability to have energy $E$ at time $t_{w}+t$, given this thermal history, is:

$$
P\left(E, t_{w}, t\right)=\int_{0}^{\infty} d E_{0} G_{\mathrm{T}_{2}}\left(E, E_{0}, t\right) P_{\mathrm{T}_{1}}\left(E_{0}, t_{w}\right)
$$

Taking the double Laplace transform with respect to $t_{w}$ and $t$ :

$$
\hat{P}\left(E, s_{w}, s\right)=\int_{0}^{\infty} d E_{0} \hat{G}_{\mathrm{T}_{2}}\left(E, E_{0}, s\right) \hat{P}_{\mathrm{T}_{1}}\left(E_{0}, s_{w}\right)
$$

Using the asymptotic expressions Eqs. (37) and (40), one can write:

$$
\hat{P}\left(E, s_{w}, s\right)=\frac{e^{\beta_{2} E}}{1+s e^{\beta_{2} E}} \hat{\Pi}_{\mathrm{T}_{1}}\left(E, s_{w}\right)+\hat{\Pi}_{\mathrm{T}_{2}}(E, s) \int_{0}^{\infty} d E_{0} \frac{\hat{\Pi}_{\mathrm{T}_{1}}\left(E_{0}, s_{w}\right)}{1+s e^{\beta_{2}} E}
$$

\section{B. Evolution of the average energy and scaling relation in the aging regime}

Bearing in mind that the energy of a given state is the opposite of the energy barrier $E$, the mean energy $\bar{E}(t)$ at time $t$ is defined by:

$$
-\bar{E}(t)=\int_{0}^{\infty} d E E P_{\mathrm{T}}(E, t)
$$

Taking into account the thermal history introduced in the preceding section, we define the energy variation between time $t_{w}$ and $t_{w}+t$ :

$$
\Delta E\left(t_{w}, t\right) \equiv \bar{E}\left(t_{w}+t\right)-\bar{E}\left(t_{w}\right)
$$

Computing the double Laplace transform yields:

$$
-\Delta \hat{E}\left(s_{w}, s\right)=\int_{0}^{\infty} d E \int_{0}^{\infty} d E_{0}\left(E-E_{0}\right) \hat{G}_{\mathrm{T}_{2}}\left(E, E_{0}, s\right) \hat{P}_{\mathrm{T}_{1}}\left(E_{0}, s_{w}\right)
$$

Expanding this equation, one finds for $s_{w} \tau_{0}$ and $s \tau_{0} \ll 1$ :

$$
\begin{aligned}
-\Delta \hat{E}\left(s_{w}, s\right) & =\int_{0}^{\infty} d E E \hat{\Pi}_{\mathrm{T}_{2}}(E, s) \int_{0}^{\infty} d E_{0} \frac{\hat{\Pi}_{\mathrm{T}_{1}}\left(E_{0}, s_{w}\right)}{1+s e^{\beta_{2} E_{0}}}-\frac{1}{s} \int_{0}^{\infty} d E_{0} E_{0} \frac{\hat{\Pi}_{\mathrm{T}_{1}}\left(E_{0}, s_{w}\right)}{1+s e^{\beta_{2} E_{0}}} \\
& =\hat{I}(s) \hat{J}\left(s_{w}, s\right)-\frac{1}{s} \hat{K}\left(s_{w}, s\right)
\end{aligned}
$$


where $\hat{I}(s), \hat{J}\left(s_{w}, s\right)$ and $\hat{K}\left(s_{w}, s\right)$ denote respectively the three integrals appearing in Eq. (47). Making the change of variable $\tau=e^{\beta_{2} E}$ in $\hat{I}$ and $\tau=e^{\beta_{1} E_{0}}$ in $\hat{J}$ and $\hat{K}$, one has:

$$
\begin{aligned}
\hat{I}(s) & =\frac{\sin \pi \theta_{2}}{\pi} \int_{1}^{\infty} d \tau \frac{T_{2} \ln \tau}{(1+s \tau)(s \tau)^{\theta_{2}}} \\
\hat{J}\left(s_{w}, s\right) & =\frac{\sin \pi \theta_{1}}{\pi} \int_{1}^{\infty} \frac{d \tau}{\left(1+s \tau^{\gamma}\right)\left(1+s_{w} \tau\right)\left(s_{w} \tau\right)^{\theta_{1}}} \\
\hat{K}\left(s_{w}, s\right) & =\frac{\sin \pi \theta_{1}}{\pi} \int_{1}^{\infty} \frac{d \tau T_{1} \ln \tau}{\left(1+s \tau^{\gamma}\right)\left(1+s_{w} \tau\right)\left(s_{w} \tau\right)^{\theta_{1}}}
\end{aligned}
$$

with $\gamma=T_{1} / T_{2} ; \hat{I}(s)$ can be computed using the identity $\ln \tau=\partial \tau^{\alpha} / \partial \alpha_{\mid \alpha=0}$; one finds:

$$
\hat{I}(s)=\frac{T_{2}}{s}\left(\pi \cot \pi \theta_{2}-\ln s\right)
$$

Let us show that $\Delta \hat{E}\left(s_{w}, s\right)$ satisfies a scaling relation. Note first that for $s \rightarrow 0, \hat{J}\left(s_{w}, s\right)$ is of the form:

$$
\hat{J}\left(s_{w}, s\right)=\frac{1}{s^{1 / \gamma}} \int_{0}^{\infty} d u \frac{f\left(s_{w} u / s^{1 / \gamma}\right)}{1+u^{\gamma}}
$$

where $f(x)=\left(\sin \pi \theta_{1}\right) /\left[\pi x^{\theta_{1}}(1+x)\right]$, and $u=s^{1 / \gamma} \tau$. In the same way, $\hat{K}\left(s_{w}, s\right)$ reads:

$$
\hat{K}\left(s_{w}, s\right)=-T_{2} \ln s \hat{J}\left(s_{w}, s\right)+\frac{T_{1}}{s^{1 / \gamma}} \int_{0}^{\infty} d u \frac{f\left(s_{w} u / s^{1 / \gamma}\right)}{1+u^{\gamma}} \ln u
$$

Coming back to $\Delta \hat{E}\left(s_{w}, s\right)$, one has from Eq. (48) that terms in ln $s$ cancel, and one gets:

$$
-\Delta \hat{E}\left(s_{w}, s\right)=\frac{1}{s^{1+1 / \gamma}}\left[\pi T_{2} \cot \pi \theta_{2} \int_{0}^{\infty} d u \frac{f\left(s_{w} u / s^{1 / \gamma}\right)}{1+u^{\gamma}}-T_{1} \int_{0}^{\infty} d u \frac{f\left(s_{w} u / s^{1 / \gamma}\right)}{1+u^{\gamma}} \ln u\right]=\frac{1}{s^{1+1 / \gamma}} \varphi\left(\frac{s_{w}}{s^{1 / \gamma}}\right)
$$

which implies a simple scaling form $\Delta E\left(t_{w}, t\right)=\psi\left(t / t_{w}^{\gamma}\right)$. This is easily shown by computing the Laplace transform of this scaling form:

$$
\mathcal{L}_{t_{w} t} \psi\left(\frac{t}{t_{w}^{\gamma}}\right)=\int_{0}^{\infty} d t \int_{0}^{\infty} d t_{w} e^{-s t} e^{-s_{w} t_{w}} \psi\left(\frac{t}{t_{w}^{\gamma}}\right)
$$

Let us make the following changes of variable: $t=x t_{w}^{\gamma}$ (at fixed $t_{w}$ ), and then $t_{w}=v /(s x)^{1 / \gamma}$ (at fixed $x$ ). One finally gets:

$$
\mathcal{L}_{t_{w}} \psi\left(\frac{t}{t_{w}^{\gamma}}\right)=\frac{1}{s^{1+1 / \gamma}} \int_{0}^{\infty} \frac{d x}{x^{1+1 / \gamma}} \psi(x) \int_{0}^{\infty} d v v^{\gamma} \exp \left(-\frac{s_{w}}{s^{1 / \gamma}} \frac{v}{x^{1 / \gamma}}-v^{\gamma}\right)=\frac{1}{s^{1+1 / \gamma}} \varphi\left(\frac{s_{w}}{s^{1 / \gamma}}\right)
$$

which indeed gives back the expected scaling form in Laplace space.

\section{Short time behaviour}

In this section, we shall focus on the short time behaviour of $\Delta E\left(t_{w}, t\right)$, characterized by $t \ll t_{w}^{\gamma}$, or equivalently $s \gg s_{w}^{\gamma}$. Note however that we consider only times that are large compared to the microscopic time scale: $t, t_{w} \gg$ $\tau_{0}=1\left(s, s_{w} \ll 1\right)$. From Eq. (55), one sees that two integrals have to be computed:

$$
\begin{aligned}
& A(\lambda)=\frac{\sin \pi \theta_{1}}{\pi} \int_{0}^{\infty} \frac{d u}{\left(1+u^{\gamma}\right)(1+\lambda u)(\lambda u)^{\theta_{1}}} \\
& B(\lambda)=\frac{\sin \pi \theta_{1}}{\pi} \int_{0}^{\infty} \frac{\ln u d u}{\left(1+u^{\gamma}\right)(1+\lambda u)(\lambda u)^{\theta_{1}}}
\end{aligned}
$$

where $\lambda$ stands for the ratio $s_{w} / s^{1 / \gamma}$. In the case $\lambda \ll 1$, these integrals reduce to: 


$$
A(\lambda)=\frac{\sin \pi \theta_{1}}{\pi \lambda^{\theta_{1}}} \int_{0}^{\infty} \frac{d u}{\left(1+u^{\gamma}\right) u^{\theta_{1}}} \quad B(\lambda)=\frac{\sin \pi \theta_{1}}{\pi \lambda^{\theta_{1}}} \int_{0}^{\infty} \frac{\ln u d u}{\left(1+u^{\gamma}\right) u^{\theta_{1}}}
$$

on condition that $\theta_{1}+\gamma>1$. The opposite case, $\theta_{1}+\gamma<1$, will be considered later on. The integrals $A(\lambda)$ and $B(\lambda)$ are readily calculated using the following identities:

$$
\int_{0}^{\infty} \frac{d v}{v^{\mu}(1+v)}=\frac{\pi}{\sin \pi \mu} \quad \int_{0}^{\infty} \frac{\ln v d v}{v^{\mu}(1+v)}=\frac{\pi^{2} \cos \pi \mu}{\sin ^{2} \pi \mu}
$$

Altogether, one finds for $\Delta \hat{E}\left(s_{w}, s\right)$ :

$$
-\Delta \hat{E}\left(s_{w}, s\right) \simeq \frac{T_{2} \pi \sin \pi \theta_{1}}{\gamma \sin \frac{\pi}{\gamma}\left(1-\theta_{1}\right)}\left[\cot \frac{\pi}{\gamma}\left(1-\theta_{1}\right)+\cot \pi \theta_{2}\right] \frac{1}{s^{1+1 / \gamma}}\left(\frac{s^{1 / \gamma}}{s_{w}}\right)^{\theta_{1}}
$$

The short time behaviour of $\Delta E\left(t_{w}, t\right)$ is obtained by inverse Laplace transform, in the case $\theta_{1}+\gamma>1$ :

$$
\Delta E\left(t_{w}, t\right) \simeq K_{>}\left(\frac{t}{t_{w}^{\gamma}}\right)^{\left(1-\theta_{1}\right) / \gamma} \quad t \ll t_{w}^{\gamma}
$$

where the coefficient $K_{>}$is given by:

$$
K_{>}=-\frac{T_{2} \pi \sin \pi \theta_{1}\left[\cot \frac{\pi}{\gamma}\left(1-\theta_{1}\right)+\cot \pi \theta_{2}\right]}{\gamma \sin \left[\frac{\pi}{\gamma}\left(1-\theta_{1}\right)\right] \Gamma\left(\theta_{1}\right) \Gamma\left(\frac{1+\gamma-\theta_{1}}{\theta_{1}}\right)}
$$

Note that in spite of the minus sign in the r.h.s. of Eq. $(63), \Delta E\left(t_{w}, t\right)$ is indeed positive at short times for $\theta_{2}>\theta_{1}$, showing that the energy has to increase first before reaching lower values. However, this coefficient vanishes for $\theta_{2}=\theta_{1}$ (i.e. no singularity occurs if temperature is kept constant), and becomes negative for $\theta_{2}<\theta_{1}$.

In the opposite case, $\theta_{1}+\gamma<1$, another approximation has to be used. Making the change of variable $v=\lambda u$ in $A(\lambda)$ and $B(\lambda)$-see Eqs. (58,59)- one finds:

$$
\begin{aligned}
& A(\lambda)=\frac{\sin \pi \theta_{1}}{\pi \lambda} \int_{0}^{\infty} \frac{d v}{\left[1+(v / \lambda)^{\gamma}\right](1+v) v^{\theta_{1}}} \\
& B(\lambda)=\frac{\sin \pi \theta_{1}}{\pi \lambda} \int_{0}^{\infty} d v \frac{\ln v-\ln \lambda}{\left[1+(v / \lambda)^{\gamma}\right](1+v) v^{\theta_{1}}}
\end{aligned}
$$

In the small $\lambda$ limit, $(v / \lambda)^{\gamma} \gg 1$, so that $A(\lambda)$ and $B(\lambda)$ reduce to:

$$
A(\lambda) \simeq \frac{\sin \pi \theta_{1}}{\pi \lambda^{1-\gamma}} \int_{0}^{\infty} \frac{d v}{v^{\gamma+\theta_{1}}(1+v)} \quad B(\lambda) \simeq \frac{\sin \pi \theta_{1}}{\pi \lambda^{1-\gamma}} \int_{0}^{\infty} d v \frac{\ln v-\ln \lambda}{v^{\gamma+\theta_{1}}(1+v)}
$$

which are indeed convergent since $\gamma+\theta_{1}<1$. One then finds for $\Delta \hat{E}\left(s_{w}, s\right)$ :

$$
-\Delta \hat{E}\left(s_{w}, s\right) \simeq \frac{\sin \pi \theta_{1}}{\sin \pi\left(\gamma+\theta_{1}\right)}\left[\pi T_{2} \cot \pi \theta_{2}-\pi T_{1} \cot \pi\left(\gamma+\theta_{1}\right)+T_{1} \ln \frac{s_{w}}{s^{1 / \gamma}}\right] \frac{1}{s^{1+1 / \gamma}}\left(\frac{s^{1 / \gamma}}{s_{w}}\right)^{1-\gamma}
$$

The inverse Laplace transform yields:

$$
\Delta E\left(t_{w}, t\right) \simeq K_{<}\left(C-\ln \frac{t}{t_{w}^{\gamma}}\right) \frac{t}{t_{w}^{\gamma}} \quad t \ll t_{w}^{\gamma}
$$

where $K_{<}$and $C$ are given by:

$$
\begin{aligned}
K_{<} & =\frac{T_{2} \sin \pi \theta_{1}}{\Gamma(1-\gamma) \sin \pi\left(\gamma+\theta_{1}\right)} \\
C & =\gamma \frac{\Gamma^{\prime}(1-\gamma)}{\Gamma(1-\gamma)}-\Gamma^{\prime}(2)+\pi \cot \pi \theta_{2}-\pi \cot \pi\left(\gamma+\theta_{1}\right)
\end{aligned}
$$




\section{Long time behaviour}

One can also study the long time behaviour $t \gg t_{w}^{\gamma}$, which happens to be easier to handle than the short time one. Coming back to the starting equation (47), the limit $s \ll s_{w}^{\gamma}$ simplifies a lot the equation, and one gets:

$$
-\Delta \hat{E}\left(s_{w}, s\right)=\frac{T_{2}}{s}\left[\pi \cot \pi \theta_{2}-\ln s\right] \underbrace{\int_{0}^{\infty} d E_{0} \hat{\Pi}_{\mathrm{T}_{1}}\left(E_{0}, s_{w}\right)}_{1 / s_{w}}-\frac{1}{s} \int_{0}^{\infty} d E_{0} E_{0} \hat{\Pi}_{\mathrm{T}_{1}}\left(E_{0}, s_{w}\right)
$$

The second term is nothing but $\mathcal{L}_{t t_{w}} \bar{E}\left(t_{w}\right)$, which also appears in the left hand side of the equation, due to the definition of $\Delta \hat{E}\left(s_{w}, s\right)$. In other words, the long time behaviour of $\bar{E}\left(t_{w}+t\right)$ appears to be the same as if the system had been quenched from high temperature to $T_{2}$ at time $t_{w}$. One finally finds:

$$
\begin{aligned}
\Delta E\left(t_{w}, t\right) & =\bar{E}\left(t_{w}+t\right)-\bar{E}\left(t_{w}\right) \\
\bar{E}\left(t_{w}+t\right) & =-\mathcal{L}_{t}^{-1} \frac{T_{2}}{s}\left(\pi \cot \pi \theta_{2}-\ln s\right) \\
& =T_{2}\left[\Gamma^{\prime}(1)-\pi \cot \pi \theta_{2}\right]-T_{2} \ln t
\end{aligned}
$$

showing that $\bar{E}\left(t_{w}+t\right)$ is indeed independent from $t_{w}$ and from $T_{1}$ for times $t \gg t_{w}^{\gamma}$. This result can also be found directly without using this particular thermal procedure: if one computes the probability distribution $P(E, t)$ for large times $t$, starting from an arbitrary initial distribution $P_{0}(E)$, it appears that the asymptotic (large $t$ ) distribution does not depend on $P_{0}(E)$ :

$$
\bar{E}\left(t_{w}+t, t_{w}\right)=\bar{E}_{\text {late }}(t)
$$

where $\bar{E}_{\text {late }}(t)$ is the average energy at a large time $t$ after a quench from high temperature.

[1] F. Lefloch, J. Hammann, M. Ocio and E. Vincent, Europhys. Lett 18, 647 (1992).

[2] K. Jonason, E. Vincent, J. Hammann, J.-P. Bouchaud and P. Nordblad, Phys. Rev. Lett. 81, 3243 (1998).

[3] J.-P. Bouchaud, V. Dupuis, J. Hammann and E. Vincent, Phys. Rev. B 65, 024439 (2001).

[4] L. Bellon, S. Ciliberto and C. Laroche, Europhys. Lett. 51, 551 (2001).

[5] J.-P. Bouchaud, P. Doussineau, T. de Lacerda-Aroso, A. Levelut, Eur. Phys. J. B 21, 335 (2001).

[6] O. Kircher, R. Bohmer, Eur. Phys. J. B 26, 329 (2002).

[7] A. Parker and V. Normand, preprint cond-mat/0306056.

[8] F. Ozon, T. Narita, A. Knaebel, G. Debregeas, P. Hebraud, J.-P. Munch, preprint cond-mat/0210554.

[9] A.J. Kovacs, Adv. Polym. Sci. (Fortschr. Hochpolym. Forsch.) 3, 394 (1963); A.J. Kovacs, J.J Aklonis, J.M. Hutchinson and A.R. Ramos, Journal of Polymer Science 17, 1097 (1979).

[10] L. Berthier, P.C.W. Holdsworth, Europhys. Lett. 58, 35 (2002).

[11] C. Josserand, A.V. Tkachenko, D.M. Mueth, H.M. Jaeger, Phys. Rev. Lett. 85, 3632 (2000).

[12] L. Berthier, J.-P. Bouchaud, Phys. Rev. B 66, 054404 (2002).

[13] S. Mossa and F. Sciortino, Kovacs effects in an aging molecular liquid, preprint cond-mat/0305526. These authors show that, although the volume is the same at the beginning and at the end of the thermal history, the visited configurations in the energy landscape are very different.

[14] F. Alberici-Kious, J.-P. Bouchaud, L. F. Cugliandolo, P. Doussineau and A. Levelut, Phys. Rev. Lett. 81, 4987 (1998).

[15] M. Sasaki, V. Dupuis, J.-P. Bouchaud, E. Vincent, Eur. Phys. J. B 29, 469 (2002).

[16] L. Berthier, V. Viasnoff, O. White, V. Orlyanchik, F. Krzakala, Proceedings of Les Houches Summer School 2002, Slow relaxations and non-equilibrium dynamics in condensed matter, J.L. Barrat, J. Kurchan, J. Dalibard Edts, EDP Sciences 2003.

[17] S. Brawer, Phys. and Chem. of Glasses 19, 48 (1978).

[18] J.-P. Bouchaud, J. Phys. I (France), 2, 1705 (1992).

[19] J.-P. Bouchaud, D.S. Dean, J. Phys. I (France) 5, 265 (1995).

[20] C. Monthus, J.-P. Bouchaud, J. Phys. A 29, 3847 (1996).

[21] P. Sollich, F. Lequeux, P. Hebraud, M. Cates, Phys. Rev. Lett. 70, 2020 (1997); P. Sollich, Phys. Rev. E 58, 738 (1998); S.M. Fielding, P. Sollich, M. Cates, J. Rheology 44, 323 (2000). 
[22] A. Kabla, G. Debregeas, preprint cond-mat/0303560.

[23] J.-P. Bouchaud, Proceedings of Les Houches Summer School 2002, Slow relaxations and non-equilibrium dynamics in condensed matter, J.L. Barrat, J. Kurchan, J. Dalibard Edts, EDP Sciences 2003.

[24] G. Ben Arous, A. Bovier, V. Gayrard, Phys. Rev. Lett. 88, 087201 (2002).

[25] R.A. Denny, D.R. Reichman, J.-P. Bouchaud, Phys. Rev. Lett. 90, 025503 (2003).

[26] M. Sasaki and K. Nemoto, J. Phys. Soc. Jpn. 69, 2642 (2000).

[27] C. Donati, J.F. Douglas, W. Kob, S.J. Plimpton, P.H. Poole and S.C. Glotzer, Phys. Rev. Lett. 80, 2338 (1998).

[28] C. Donati, S.C. Glotzer, P.H. Poole, W. Kob and S.J. Plimpton, preprint cond-mat/9810060.

[29] E.R. Weeks, J.C. Crocker, A.C. Levitt, A. Schofield, D.A. Weitz, Science 287, 627 (2000).

[30] B. Doliwa, A. Heuer, J. Phys. Condens. Mat. 15 (Sp. Iss.), s849 (2003).

[31] L. Berthier, Phys. Rev. Lett. 91, 055701 (2003).

[32] C. Toninelli, G. Biroli and D.S. Fisher, preprint cond-mat/0306746.

[33] L. Berthier, J.-P. Garrahan, J. Chem. Phys. 199, 4367 (2003).

[34] J.-P. Bouchaud, V. Dupuis, J. Hammann, E. Vincent, Phys. Rev. B 65, 024439 (2002).

[35] J.-P. Bouchaud, L. Cugliandolo, J. Kurchan, M. Mézard, in Spin-glasses and Random Fields, edited by A. P. Young (World Scientific, Singapore, 1998), and references therein. 